Mitteilungen der Deutschen Gesellschaft für Phlebologie

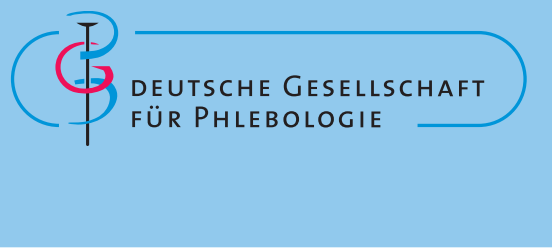

\title{
Jahrestagung der DGP in Aachen auch im Livestream
}

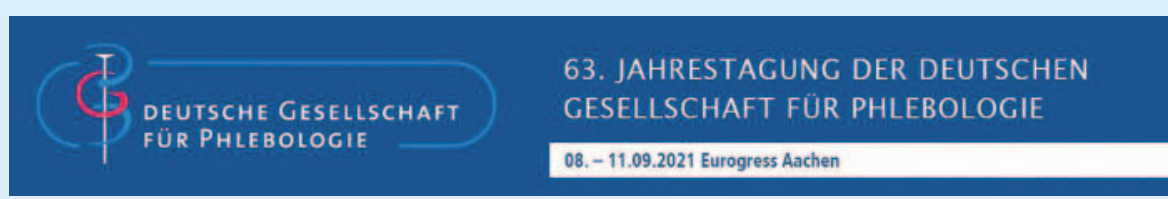

Die Jahrestagung der Deutschen Gesellschaft für Phlebologie in Aachen vom 8.-11.09.2021 steht vor der Tür.

Das vergangene Jahr hat gezeigt, wie wichtig das persönliche Miteinander ist. Wir sind weiterhin sehr optimistisch, dass die Lage es erlauben wird, sich zur DGP-Jahrestagung im Eurogress in Aachen in Präsenz treffen zu können.

Die Programmeinzelheiten finden Sie auf der Website: https://phlebologie-2021.de.

Wie kaum ein anderes Teilgebiet in der Medizin ist die Phlebologie geprägt von der interdisziplinären und interprofessionellen Zusammenarbeit in Forschung und Krankenversorgung. Mehr noch, sie lebt vom Zusammenwirken der unterschiedlichen Fachgruppen. Daher steht die kommende Jahrestagung auch unter dem Motto "Synergie und Vielfalt“. Dabei greifen wir auf Bewährtes der vergangenen Kongresse zurück und haben zudem durch wissenschaftliche Impulse weltweiter Experten ein für unsere Gäste und Teilnehmer attraktives Programm gestaltet.

Inhaltlich werden die klassischen Themen der Phlebologie mit den Erkrankungen des oberflächlichen und tiefen Beinvenensystems, den chronischen Wunden, dem Lip- und Lymphödem und den Gefäßmalformationen behandelt.

Darüber hinaus soll der venösen Forschung ebenso wie der Ästhetik in der Phlebologie Raum in wissenschaftlichen Sitzungen gegeben werden. Erfolgreiche Programmpunkte der letzten Kongresse werden fortgeführt, wie beispielsweise das ganztägige ICW-zertifizierte Update Wundmanagement, der Crashkurs und das Update Phlebologie, eine umfangreiche Auswahl an Hands-on-Kursen, das Weiterbildungsforum und die für den Technologietransfer wichtigen Firmensymposien.

Neu sind in diesem Jahr neben speziellen Workshops für die Behandlung der Pathologien des tiefen Venensystems der DEGUMAufbaukurs Sonografie Haut, Subkutis und Lymphknoten, ganztägig am Samstag, und der Weiterbildungspfad, der nicht nur „Jung-Phlebologen“ die strukturierte Teilnahme von Workshops und theoretischen Programmpunkten über den gesamten Tagungszeitraum ermöglicht, um einen kompakten Überblick über die gesamte Breite des Fachs zu erhalten.

Neben der Präsenzveranstaltung bieten wir auch in diesem Jahr wieder einen Livestream an, der denjenigen Kolleginnen und Kollegen die Möglichkeit gibt, die nicht nach Aachen reisen können, am wis- senschaftlichen Programm teilzunehmen. Nutzen Sie hier die Gelegenheit zur Anmeldung https://mi.wikonect.de/online/ index?webid=DGPhlebo21A-Live.

Eingebettet ist der Kongress in ein besonders attraktives Umfeld. Aachen ist als westlichste deutsche Großstadt verkehrstechnisch hervorragend angebunden. Es besteht durch die geografische Lage im Dreiländereck der Euregio „im Herzen Europas“ eine enge Vernetzung mit unseren Nachbarländern mit unzähligen Synergien auf wissenschaftlicher, sozialer und kultureller Ebene. Aachen ist ein Ort der Vielfalt und blickt auf eine lange Tradition und Geschichte zurück, geprägt durch das Nebeneinander historischer und moderner Architektur, die sich aus der Kaiserzeit Karls des Großen bis in die Gegenwart eines erfolgreichen Technologiestandortes entwickelt hat.

Unsere Jahrestagung findet im Eurogress Aachen statt - ein idealer Rahmen für den wissenschaftlichen Transfer und den intensiven internationalen Austausch, um sich gemeinsam in einem historischen, kulturell vielseitigen und familiären Ambiente auf den neuesten Stand der Phlebologie zu bringen.

Es ist uns eine außerordentlich große Freude, Sie im September 2021 in Aachen begrüßen zu dürfen.

PD Dr. med. Knuth Rass

PD Dr. med. Houman Jalaie 